\title{
DEVELOPMENT OF TIME COUPLINGS METHOD USING EVOLUTIONARY ALGORITHMS
}

\author{
Magdalena Rogalska \\ Lublin University of Technology \\ ul. Nadbystrzycka 40, 20-950 Lublin, Poland \\ e-mail: rogalska@akropolis.pol.lublin.pl
}

\author{
Wojciech Bożejko, Zdzisław Hejducki \\ Wrocław University of Technology \\ Wybrzeże Wyspiańskiego 27, 50-370 Wrocław, \\ Poland, e-mail: wojciech.bozejko@pwr.wroc.pl
}

\author{
Mieczysław Wodecki \\ Institute of Computer Science, University of Wrocław \\ Juliot-Curie 15, 50-383 Wrocław, Poland. \\ e-mail: mwd@ii.uni.wroc.pl
}

\begin{abstract}
The article presents the results of a computation experiment in which a genetic algorithm (GA) and a hybrid evolutionary algorithm (HEA) were used. The respective results are compared for an objective function describing employment level regularity. It has been demonstrated that evolutionary algorithms can be used for optimizing demand for resources (workers) in time coupling methods.
\end{abstract}

\section{KEYWORDS}

evolutionary algorithm, genetic algorithm, time coupling method TCM

\section{INTRODUCTION}

The article presents in a concise way the Time Couplings Method (TCM) devised by Victor A. Afanasjev[1]. The main aim is to acquaint the general public of scientists in the world with TCM. So far this subject has been presented in an academic way, which made its comprehension difficult and constituted a barrier for a larger readership. In contrast, TCM computations performed using the ORGANIZATOR program are simple and quick. Considering the lack of international publications on
TCM, we present the general idea of the method, the stages in its development and the way of computing the main. A calculation example is provided. The current development of TCM with regard to new applications is described.

Linear scheduling methods (LSM), referred to as TCM (Time Couplings Method) in Central and East European Countries, stem from the course of construction work, based on the division of a project into sectors. Because of the nature of construction work, the two varieties of linear scheduling methods 
have been developing in parallel despite the iron curtain between the East of Europe and the rest of the world. It is precisely the natural connection between practical methods and their scientific description that caused the parallel development of the methods in Russia and Poland and in such countries as Canada and the USA.One of the followers of M.S. Budnikov (1961) was V.A. Afanasjev $(1957,1988,2000)$ who in a scientific way described work carried out in accordance with LSM, assuming sectors of different size and so different task realization times for particular gangs. All the nesessary calculations could be done manually but they were laborious and time-consuming.In the late 1970 s the methods were adapted for the use on RIAD computers. Juliusz Mrozowicz (1982) was the first one to propagate LSM in Poland. He introduced a LSM systematics in Polish and presented LSM assumptions, terminology and calculation methods. Zdzisław Hejducki (1999,2000, 2001,2003, 2004) improved LSM by introducing task optimisation and reduction. He described in a scientific way the process commencement time, duration and completion time computing algorithms for different constraints.

Scheduling of construction projects by the Time Couplings Method (time couplings are interdependences between activities and sectors, which take resource and technical limitations into account), first developed by Victor A. Afanasjev and applied by him to a military airfield construction project in the USSR in 1946, has not been presented internationally because of the Soviet Union's separatistic policies and secretiveness and because this was not required by that country's scientific community. Despite its numerous applications in Eastern Europe the method is unknown in the world. Due to the lack of a basic publication which would explain the method's fundamentals, papers describing its advanced aspects cannot be published in international journals.

TCM's main advantage is that time couplings (not only total project time TT but also all the interconnections) can be computed using the ORGANIZATOR software. The drawbacks are that only one contractor can work in one sector at the same time and the ORGANIZATOR program's capacity is limited to 20 sectors and 20 activities. It is, however, possible to combine two or more computing sequences.

The optimization problem is as follows: for an assigned construction project lead time the best (according to the adopted optimization criterion) employment level must be found. One of the optimization criteria can be to include the average demand for resources in the constructed objective function. In order to search for a minimum demand one can adopt the average deviation from the daily demand as a measure of the unevenness of the demand for workers.

In the West, linear scheduling methods have developed mainly in Canada and the USA. In (1969) O'Brien introduces the notion of 'Line of Balance'. The subject is also investigated by Carr and Meyer (1974) and Halpin and Woodhead (1976). Peer(1974) and Selinger (1980), Handa and Barcia (1986) and Chrzanowski and Johnston (1986) develop a 'Construction Planning Technique', O'Brien (1975) and Barrie and Paulston(1978) - a 'Vertical Production Method', Birrell (1980) - a 'Time-Location Matrix Model', Johnston (1981) and Stradl and Cacha (1982) - a 'Time Space Scheduling Method'. Whiteman and Irwig (1988) publish works on 'Disturbance Scheduling' and Thabet and Beliveau (1994) - on 'Horizontal and Vertical Logic Scheduling for Multistory Projects. O’Brien (1975, 1985), Arditi and Albulak (1979, 1986) and Melin and Whiteaker(1981) develop linear scheduling tools such as: line-of-balance diagrams, bar-charts, vertical production charts and velocity diagrams. Russel and Caselton (1988) introduce extensions to linear scheduling optimisation, having the form of interrupt duration vector choices. Russel and Wong (1993) describe the development and use of a new generation of planning structures providing a basis for a superset for the traditional critical path method and over the years introduce various extensions needed to represent the realities of construction. Senouci and Eldin (1996) present a nonserial dynamic programming formulation for the scheduling of linear projects with nonsequential activities. El-Rayes and Moselhi (1998, 2001) optimise resources in repetitive construction projects. Harris and Ioannou (1998) work out a 
repetitive scheduling method with technical constraints. Adeli and Karim (1997) develop a neural dynamics cost optimisation model for construction projects.

In contradistinction to the above methods, in TCM the way in which the project is to be carried out: method 1 - no work stoppages, method 2 - no stoppages in sectors, method 3 - minimum lead time and possible work and sector stoppages, methods 4, 5, 6 minimum time and additional constraints is assumed for the calculations. Currently research focuses on hybrid methods of optimising complex construction processes. Packages of processes described by TCM, LSM, LOB, genetic algorithms, neural networks and bar-charts are incorporated into one schedule. This work is the continuation of author's research on constructing efficient algorithms to solve hard problems of management which can be applied in construction (Bożejko and Wodecki (2005) .

\section{PRINCIPLES OF TCM APPLICATION}

As described in [1], in order to begin scheduling by TCM one should have:

- input data specifying the division of a project into sectors $\mathrm{S} 1$.....Sn (a sector is a part of land or building, a fragment of a pipeline, etc.) and the size of the latter,

- $\quad$ specified kinds of activities and the technological sequence (determined by the technology used) in which they are to be carried out,

- deterministically fixed (determined on the basis of catalogue data which in Poland are collected in the Catalogue of Labour Standards) times of performing the activities in each sector.

As a result of TCM computations the following are obtained:

- a work schedule,

- networks of dependences,

- a table of internal dependences between the start and finish times of the particular activities in the sectors.

\section{COMPUTATION EXPERIMENT OF TCM - CASE STUDY}

The numerical data for the optimization calculations are for a complex of 12 building structures on which 9 construction processes are to be carried out. The project is represented by a matrix of construction process $(r=9)$ durations for the structures $(f=12)$. The work duration matrix elements were based on bills of quantities for a housing complex. Time coupling method TCM III was used to determine the construction project cycle $(\mathrm{T}=216$ working days). The method allows one to identify a sequence of critical processes and fix noncritical work commencement and completion dates. Owing to the changeable position of the jobs along the time axis one can optimize the employment level according to the adopted objective function.

The optimization problem is as follows: for an assigned construction project lead time the best (according to the adopted optimization criterion) employment level must be found. One of the optimization criteria can be to include the average demand for resources in the constructed objective function. In order to search for a minimum demand one can adopt the average deviation from the daily demand as a measure of the unevenness of the demand for workers.

$$
\mathbf{T}=\left[\begin{array}{cccccccccccc}
7 & 8 & 7 & 7 & 7 & 8 & 7 & 7 & 6 & 7 & 5 & 4 \\
8 & 11 & 8 & 9 & 9 & 11 & 8 & 9 & 8 & 9 & 8 & 8 \\
8 & 11 & 10 & 9 & 9 & 11 & 10 & 9 & 11 & 9 & 9 & 9 \\
7 & 8 & 7 & 7 & 8 & 8 & 7 & 7 & 8 & 8 & 8 & 7 \\
13 & 6 & 7 & 7 & 7 & 7 & 7 & 7 & 7 & 7 & 8 & 15 \\
11 & 14 & 11 & 13 & 13 & 14 & 11 & 13 & 14 & 13 & 14 & 8 \\
9 & 14 & 9 & 11 & 10 & 13 & 9 & 11 & 8 & 10 & 11 & 9 \\
4 & 8 & 6 & 7 & 5 & 7 & 7 & 8 & 9 & 9 & 9 & 5 \\
6 & 9 & 5 & 9 & 7 & 5 & 8 & 9 & 8 & 7 & 7 & 7
\end{array}\right]
$$

The objective function

$$
f(x)=\frac{1}{T} \sum_{j=1}^{T}\left|\frac{q_{j}(x)-r_{a v g}}{r_{a n g}}\right|
$$

where: 
$r_{\text {avg }}=\frac{1}{T} \sum_{i=1}^{n} d_{i} r_{i}$

the average number of workers employed on each of the $T$ days.

$x \in R^{n}$

$x=\left(x_{1}, x_{2}, \ldots, x_{n}\right)-$ the vector of the tasks' starting times,

$\mathrm{x}_{\mathrm{i}} \in\left[\mathrm{a}_{\mathrm{i}}, \mathrm{b}_{\mathrm{i}}\right]$

$a_{i}$ - the earliest time of starting task $i$,

$b_{i}$ - the latest time of starting task $i$,

$q_{j}(x)$ - the number of persons employed on day $j, j=$ $1,2, \ldots, T, T-$ a time horizon,

$d_{i}-$ duration of process $i$

$r_{i}$ - the number of persons employed to carry out process $i$.

In order to carry out optimization calculations one must calculate the objective function for each iteration. The function can be calculated as the average percentage deviation of the daily number of employed persons from the average employment during all the $T$ days, i.e. $f(x) \in[0,1]$ for a standard objective function.

\section{BASIC PROPERTIES OF HYBRID EVOLUTIONARY ALGORITHM}

The algorithm [7] starts with the (random) creation of initial population $P^{0}$. The best member of population $P^{0}$ is adopted as suboptimal solution $\pi^{*}$. Let $i$ be an algorithm iteration number. New population $i+1$ (i.e. set $P^{i+1}$ ) is generated as follows. For current population $P^{i}$ a set of local minima $\left(L M^{i}\right)$ is fixed (by carrying out the
$\operatorname{LocalOpt}(\pi)$ procedure for each element $\pi \in P^{i}$ ). Elements which occur in the same positions at the local minima are fixed (procedure FixeSet $\left.\left(L M^{i}, F S^{i}\right)\right)$ and a set of fixed elements and positions $\left(F S^{i+1}\right)$ is created. Each permutation of new population $P^{i+1}$ has fixed elements (in fixed positions) from set $F S^{i+1}$. Free elements are randomly assigned to the remaining (free) positions. If permutations $\beta \in L M^{i}$ and $F(\beta)<F\left(\pi^{*}\right)$ exist, then $\beta$ is adopted for permutation $\pi^{*}$. The algorithm ends after a predetermined number of generations have been generated.

\section{Hybrid Evolutionary Algorithm (HEA)}

Initialization: randomly created population $P^{0}=\left\{\pi_{1}, \pi_{2}, \ldots, \pi_{\eta}\right\}$;

$\pi^{*}=$ the best member of population $P^{0}$;

the number of iteration $i=0 ; \quad F S^{0}=\varnothing$;

\section{repeat}

Fix local minima set

$L M^{i}=\left\{\hat{\pi}_{1}, \hat{\pi}_{2}, \ldots, \hat{\pi}_{\eta}\right\}$, where

$\hat{\pi}_{j}=\operatorname{LocalOpt}\left(\pi_{j}\right), \pi_{j} \in P^{i} ;$

for $j:=1$ to $\eta \underline{\text { do }}$ if

$F\left(\hat{\pi}_{j}\right)<F\left(\pi^{*}\right)$ then $\pi^{*} \leftarrow \hat{\pi}_{j}$;

Fix set

$F S^{i+1}=F i x \operatorname{Set}\left(L M^{i}, F S^{i}\right)$ and

generate new population

$P^{i+1}:=\operatorname{NewPopulation}\left(F S^{i}\right)$;

$i=i+1$;

until not StopCriterion; 


\section{Comparison of the algorithms' properties}

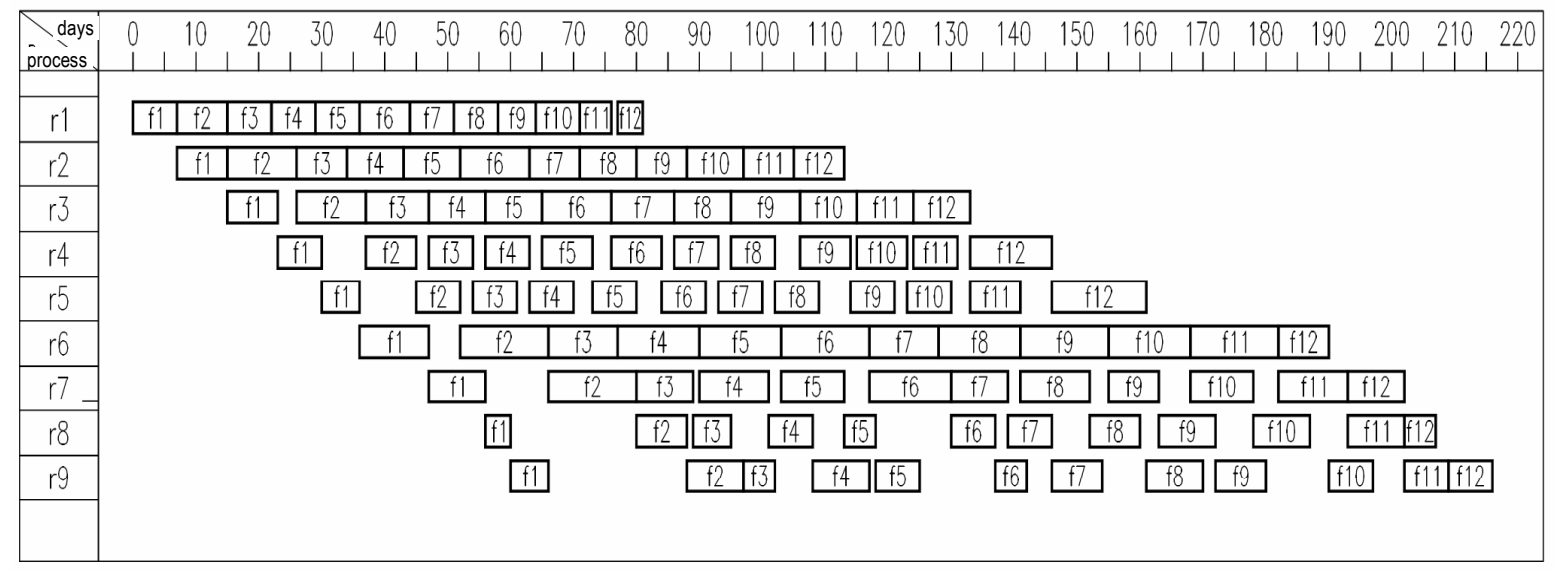

Figure 1. Gantt chart after evolutionary algorithm application, minimum objective function $f=1508.139$.

\begin{tabular}{|c|c|}
\hline $\begin{array}{c}\text { days } \\
\text { ocess } \\
\end{array}$ & 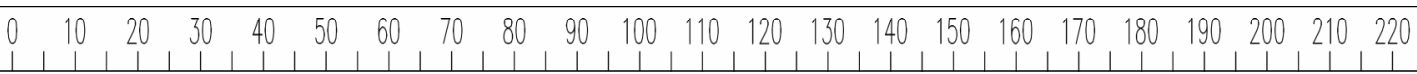 \\
\hline rl & \begin{tabular}{|l|l|l|l|l|l|l|l|l|l|}
$f 1$ & $f 2$ & $f 3$ & $f 4$ & $f 5$ & $f 6$ & $f 7$ & $f 8$ & $f 9$ & $f 10|f 11| f 10$ \\
\end{tabular} \\
\hline r2 & \begin{tabular}{|l|l|l|l|l|l|l|l|l|l|l|l|}
$f 1$ & $f 2$ & $f 3$ & $f 4$ & $f 5$ & $f 6$ & $f 7$ & $f 8$ & $f 9$ & $f 10$ & $f 11$ & $f 12$ \\
\end{tabular} \\
\hline r3 & \begin{tabular}{|l|l|l|l|l|l|l|l|l|l|l|l|}
$f 1$ & $f 2$ & $f 3$ & $f 4$ & $f 5$ & $f 6$ & $f 7$ & $f 8$ & $f 9$ & $f 10$ & $f 11$ & $f 12$ \\
\end{tabular} \\
\hline r4 & 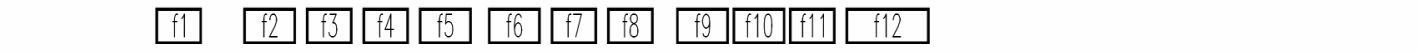 \\
\hline $\mathrm{r} 5$ & 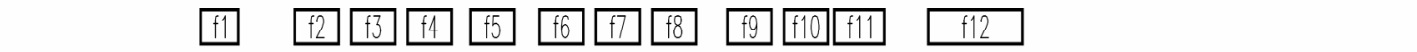 \\
\hline r6 & \begin{tabular}{|l|l|l|l|l|l|l|l|l|l|l|l|}
$f 1$ & $f 2$ & $f 3$ & $f 4$ & $f 5$ & $f 6$ & $f 7$ & $f 8$ & $f 9$ & $f 10$ & $f 11$ & $f 12$ \\
\end{tabular} \\
\hline r7 & 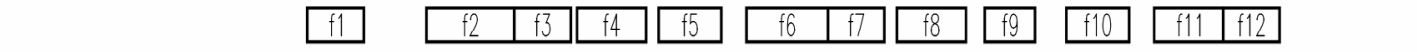 \\
\hline 18 & 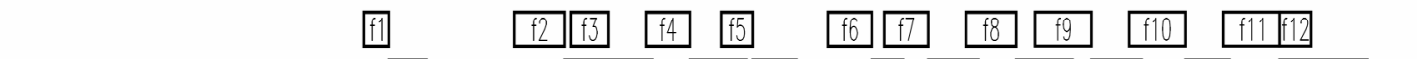 \\
\hline r9 & 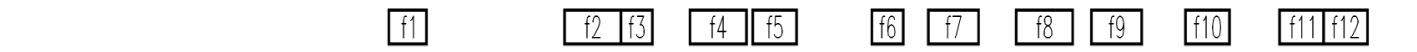 \\
\hline
\end{tabular}

Figure2. Gantt chart after genetic algorithm application, minimum objective function $f=1564.999$

\section{CONCLUSION}

An optimization problem $(12 * 9, T=216$ working days) concerning employment level planning for an assigned construction project lead time was computed using a genetic algorithm (GA) and a hybrid evolutionary algorithm (HEA) [2,3]. After 100000 iterations using GA, objective function $f=1564.999$ and the average percentage deviation of the daily number of employed persons from the average employment for all the $T$ days amounting to
0.312375 were obtained (Figure 2). After 100000 iterations using HEA, the objective function was $f=1508.139$ and the average deviation amounted to 0.301198 (Figure 1). Thanks to the use of the evolutionary algorithm the result improved by $3.57 \%$.

It has been demonstrated that evolutionary algorithms [4,5,6,7] can be used for optimizing demand for resources (workers) in time coupling methods (TCM)[1]. It is also possible to employ 
metaheuristic (simulated annealing and taboo search) algorithms and evolutionary algorithms to solve task scheduling problems in TCM. The research into this possibility is underway.

\section{REFERENCES}

[1] Afanasjev, V.A., Afanasjev A.V. (2000), "Stream methods in construction work", Sankt-Petersburg.

[2] Bożejko W., Wodecki M., (2005). A hybrid evolutionary algorithm for some discrete optimization problems. IEEE Computer Society, P2286 ISBN 0-7695-2286-6.

[3] Rogalska M., Bożejko W. Hejducki Z., (2005). Employment level control using genetic algorithms (in Polish). 51st KILiW PAN and KN PZITB Scientific Conference Gdańsk-Krynica, pp.185-192.
[4] LEU, S.S., YANG, C.H., HUANG J.C. (2000) Resource levelling in construction by genetic algorithm-based optimization and its decision support system application. Automation in Construction 10, 27-41.

[5] Otterloo van Siuwert, Evolutionary algorithms and Scheduling Problems. Universiteit Utrecht, 2002.

[6] Reeves C.R. Yamada T., (1998). Genetic Algorithms Path Relinking on the Flowshop Sequencing Problem. Evolutionary Computation Journal, MIT Press, Vol. 6., No 1, pp 230-234.

[7] Yamada T., Solving the Csum Permutation Flowshop Scheduling Problem by Genetic Local Search ICEC98 (International Conference on Evolutionary Computation), pp. 230-234. 